\title{
Ergonomics Design on Expert Convenience of Voice-Based Interface for Vehicle's AV Systems
}

\author{
Pei-Ying $\mathrm{Ku}^{1}$, Sheue-Ling Hwang ${ }^{1}$ \\ Hsin-Chang Chang ${ }^{2, *}$, Jian-Yung Hung ${ }^{2}$, and Chih-Chung Kuo ${ }^{2}$ \\ ${ }^{1}$ National Tsing Hua University, Hsinchu, Taiwan 300 \\ a60029@yahoo.com.tw \\ ${ }^{2}$ Industrial Technology Research Institute, Hsinchu, Taiwan 310 \\ piosn@itri.org.tw
}

\begin{abstract}
This research aimed to investigate and explore expert user interface design principle in adaptive user interface of in-vehicle full voiced-based interface. In this study, 3 stages of driving simulation experiments were established. The voice-based interface called Talking Car novice interface has been designed before. Through driving simulation experiments, subjects' behavior and response data when using voice-based interface were collected and analyzed. According to the result, the length of speech by Talking Car novice interface would be adjusted to fulfill expert users' requirements, and then switched to Talking Car expert interface. After that, a driving simulation experiment is conducted to verify the usability of the adapted interface as well as the implications on operation efficiency and traffic safety.
\end{abstract}

Keywords: in-vehicle full voice-based interface, Talking Car, driving simulation, expert user interface.

\section{$1 \quad$ Introduction}

\subsection{Background and Motivation}

Ministry of Transportation and Communications in Taiwan has amended 89th and 90th road safety traffic rules, indicating that drivers have to turn off all entertaining monitors within their eyesight, but not included the display of driving aided function. (Ministry of Transportation and Communications in Taiwan, 2011)

As we know, there is noticeable importance on developing an in-vehicle user interface by full voiced control. In other words, the drivers only depend on their auditory memory to use the interface, and thus it might be more difficult to teach novice users how to use the in-vehicle full voiced-based interface. On the other hand, the interface with convenience for expert users is also required. Therefore, in order to make the invehicle full voiced-based interface to be provided with novice elicitation and expert convenience, it's necessary to design an adaptive user interface.

\footnotetext{
Corresponding author.
} 
Regarding adaptive user interface in Human-Machine Interface, there are abundance of previous literatures on visual interface; however, there is little specific guidance on in-vehicle full voice-based interface. According to Jason (2010), novice users are generally concerned with how to do things instead of how fast they can do it. For this reason, novice user interface design must have complete aided functions to help novice user operating the interface step by step. Meanwhile, expert users are goal orientated and when using a user interface, they quickly deduce goals and actions to achieve those goals. They want a highly efficient interface and would thus like the number of interactions to be reduced. Hence, expert user interface design should reduce guides, and let users use the interface at their own choice to improve users' operation efficiency and traffic safety.

\subsection{Objectives}

In-Vehicle Speech Systems have been developed, and there is one (LUXGEN Think+) in-vehicle control systems in Taiwan. As a matter of fact, drivers need to use hands and eyes to complete the tasks to control systems in a vehicle. Distraction is easily brought up when drivers have to operate the control system while driving.

However, as things go, there has not been an in-vehicle speech control system with adaptive user interface. This study aimed to find the length of sentence the expert could tolerate in Talking Car, and then an In-Vehicle Speech Expert Interface with convenience was designed according to the experiment results. Furthermore, a follow up experiment was to ensure sufficient users' operation efficiency and a safe response to take care the road situations when controlling the AV System in-vehicle.

\section{Research Method}

\subsection{Experimental Design}

The Voice-based interaction interface with expert convenience was developed step by step through three stages of driving simulate experiments. This research chose music and radio functions which are commonly used by users to design the experimental tasks. The experiments include two independent variables - Talking Car users' familiarity and Talking Car's mode with novice, advanced-novice and expert. In driving simulated experiments, a camera was set to capture the subject's operations; we measure reaction time of the brakes when "STOP!!" message showed on the display, system speech times and words used by subjects using interrupt control button and the task completion time while carrying out the driving task. After the experiment finished, subjects would be asked to complete three questionnaires, included NASATLX, subjective and impatient-degree questionnaire to evaluation subjects' workload, satisfaction and the degree of impatient.

\subsection{Experimental Procedure}

Sixteen participants, including 9 males and 7 females, are students from National Tsing Hua University in different majors, and ages range from 22 to 30. Subjects have 
to hold the driving license for at least 1 year and have a habit of listening to radio or music while driving. None of them has experience of using In-vehicle speech interaction system. The following sub-section describes the testing process.

1. The process of experiment took around 1 hour in the first stage, 30 minutes in the second and the third ones.

2. Learning time took 10 minutes to introduce the system and practice to drive on the simulator.

3. Complete tasks took 15 to 20 minutes in the first stage, 5 to 10 minutes in the second and the third stages.

4. All tasks will be executed 2 times.

\section{Experimental Results}

\subsection{Suitable Sentence Length for Expert Users Analysis}

Among 15 types of sentences in Talking Car, 4 types of sentences were used more often. Therefore, the following were analyses for the 4 sentences (sentence 2, sentence 3 , sentence 5 and sentence 13). To find out the sentence length that expert users could tolerate, we recorded the number of characters (Chinese characters) used in each sentence before subjects interrupted, and then calculated the accumulated percentage of interrupted times. We then identified whether the accumulated percentage of interrupted times was within $60 \%$ or there was a huge gap between the percentage, and used the number of characters in each sentence as an indicator for the next stage of experiment. The limitation of words in each sentence length is shown in Table 1. According to the result, the speech sentence was designed to fulfill Talking Car expert interface users.

Table 1. Result of limitation of sentence length from experiments. (number of characters)

\begin{tabular}{|c|c|c|c|c|}
\hline & Sentence 2 & Sentence 3 & Sentence 5 & Sentence 13 \\
\hline First Stage & 16 & 13 & 17 & 19 \\
\hline Second Stage & 7 & 5 & 5 & 5 \\
\hline Third Stage & 7 & 5 & 5 & 5 \\
\hline
\end{tabular}

\subsection{The Suitable Timing of Interface Switch Moment}

This section would analyse the suitable timing of changing the interface from novice to expert by system automatic switch. Base on Table 1 , the limitation of sentence length could be known. In addition, we calculated the average of number of characters in each sentence used by subjects in every interrupt times; the result was shown in Table 2 and Table 3. Accordingly, we defined that novice interface (used in the first stage of experiment) could be changed into advanced-novice interface (used in the second stage of experiment) when novice users interrupted the sentence for 6 times, and advanced-novice interface could be changed into expert interface (used in the third stage of experiment) when advanced-novice interface users interrupted the sentence for 5 times. Especially, the interface was changed sentence by sentence rather 
than changed all the sentences once. In this manner, it could avoid the confusing situation that users listening the expert sentences without guidance.

Table 2. Average of interrupt moment in the first stage of experiment (number of characters)

\begin{tabular}{|c|c|c|c|c|c|c|}
\hline Interrupt Times & $\mathbf{1}$ & $\mathbf{2}$ & $\mathbf{3}$ & $\mathbf{4}$ & $\mathbf{5}$ & $\mathbf{6}$ \\
\hline Sentence 2 & 19.63 & 17.38 & $\mathbf{1 5 . 5 6}$ & 14.00 & 11.38 & 12.06 \\
\hline Sentence 3 & 16.44 & 15.44 & 17.00 & 14.38 & 13.31 & $\mathbf{1 2 . 4 4}$ \\
\hline Sentence 5 & 30.19 & 26.69 & 19.94 & 20.44 & 22.50 & $\mathbf{1 4 . 8 1}$ \\
\hline Sentence 13 & 24.88 & 21.75 & 20.56 & 17.69 & 20.25 & $\mathbf{1 6 . 4 4}$ \\
\hline
\end{tabular}

Table 3. Average of interrupt moment in the second stage of experiment (number of characters)

\begin{tabular}{|c|c|c|c|c|c|}
\hline Interrupt Times & $\mathbf{1}$ & $\mathbf{2}$ & $\mathbf{3}$ & $\mathbf{4}$ & $\mathbf{5}$ \\
\hline Sentence 2 & 7.44 & 6.81 & 6.25 & $\mathbf{6 . 6 3}$ & \\
\hline Sentence 3 & 8.94 & 8.25 & 7.25 & 8.19 & $\mathbf{6 . 6}$ \\
\hline Sentence 5 & 7.69 & 6.31 & 5.5 & 6.44 & $\mathbf{6 . 5 6}$ \\
\hline Sentence 13 & 6.88 & 6.75 & 7.56 & 7.5 & $\mathbf{5 . 5 6}$ \\
\hline
\end{tabular}

\subsection{Impatient Degree of Speech Sentence}

In these experiments, we would like to know whether the length or content of speech sentence made users irritable. Thus the subjects required to rate the degree of impatient on 15 speech sentences, including speech length and content. The rating scale was from 1 to 5 , and the degree of 5 meant that the subject felt the most impatient on the sentence. According to Table 4 and Table 5, the degree of impatient on sentence length between novice and advanced-novice interface $(\mathrm{p}>0.05)$ was not significantly difference, and the same result on impatient degree of sentence content. Between advanced-novice and expert interface, there are significant difference both on the impatient degree of sentence length and content. Moreover, we could conclude that the speech sentence on Talking Car expert interface resulted in users the lowest impatient significantly.

Table 4. Paired t-test results of impatient degree of speech sentence length

\begin{tabular}{|c|c|c|c|c|}
\hline & First Stage & Second Stage & Second Stage & Third Stage \\
\hline Mean & 2.659 & 2.583 & 2.583 & 1.876 \\
\hline P-Value & \multicolumn{2}{|c|}{0.490} & \multicolumn{2}{c|}{$0.005^{*}$} \\
\hline
\end{tabular}

$* \mathrm{p}<0.05$

Table 5. Paired t-test results of impatient degree of speech sentence content

\begin{tabular}{|c|c|c|c|c|}
\hline & First Stage & Second Stage & Second Stage & Third Stage \\
\hline Mean & 2.546 & 2.563 & 2.563 & 1.771 \\
\hline P-Value & \multicolumn{2}{|c|}{0.850} & \multicolumn{2}{c|}{$0.001 * *$} \\
\hline$* * \mathrm{p}<0.01$
\end{tabular}




\subsection{Task Completion Time}

The completion time of radio and music task was analyzed by Paired t-test. As shown in Tables 6 and 7, there were significant differences on all the tasks completion time. Moreover, the average operation time of Talking Car expert interface (third stage) was the shortest one in all interfaces due to the shortest speech sentence. It implies that expert interface has comparative convenience among these interfaces.

Table 6. Paired t-test results of task completion time on radio task

\begin{tabular}{|c|c|c|c|c|}
\hline & First Stage & Second Stage & Second Stage & Third Stage \\
\hline Mean $(\mathrm{Sec})$ & 359.84 & 127.78 & 127.78 & 102.75 \\
\hline P-Value & \multicolumn{2}{|c|}{$<0.001 * * *$} & \multicolumn{2}{c|}{$<0.001 * * *$} \\
\hline
\end{tabular}

Table 7. Paired t-test results of task completion time on music task

\begin{tabular}{|c|c|c|c|c|}
\hline & First Stage & Second Stage & Second Stage & Third Stage \\
\hline Mean $(\mathrm{Sec})$ & 187.13 & 95.22 & 95.22 & 79.44 \\
\hline P-Value & \multicolumn{2}{|c|}{$0.000 * * *$} & \multicolumn{2}{c|}{$<0.001 * * *$} \\
\hline
\end{tabular}

\subsection{Reaction Time}

Subjects' reaction time is recorded by simulated software. The calculation method of reaction time is started from the "STOP!!!" message showing on the display till subject stepping the brake to stop counting. Analyzing the average reaction time with radio and music task and without task, the result showed that there was no significant difference between "talking" task and without "talking" task in the first stage, but was significant in the second stage $(\mathrm{p}<0.001)$. Furthermore, the result also showed that the reaction times of radio and music task between the second and the third stage was not significantly different.

\subsection{NASA-TLX}

After the simulation experiment, subjects required to answer a NASA-TLX questionnaire. As shown in Table 8, the Talking Car expert interface has the lowest mental workload. The results of Paired t-test indicated that mental workload of advancednovice interface was significantly lower than that of novice interface $(p<0.01)$, but there was no significant difference between mental workload of advanced-novice and that of expert interface. It implies that Talking Car expert interface provides a more efficient user interface to the driver so that the driver can operate the Talking Car System with less mental workload. 
Table 8. Average weighted score of NASA-TLX

\begin{tabular}{|c|c|c|c|c|c|c|c|}
\hline \multicolumn{7}{|c|}{ NASA-TLX weighted score } \\
\hline Items & $\begin{array}{c}\text { Mental } \\
\text { Demands }\end{array}$ & $\begin{array}{c}\text { Physical } \\
\text { Demands }\end{array}$ & $\begin{array}{c}\text { Temporal } \\
\text { Demands }\end{array}$ & $\begin{array}{c}\text { Own } \\
\text { Performance }\end{array}$ & Effort & Frustration & Total \\
\hline First Stage & 16.77 & 6.38 & 7.63 & 8.56 & 16.90 & 5.81 & 62.05 \\
\hline $\begin{array}{c}\text { Second } \\
\text { Stage }\end{array}$ & 14.14 & 6.14 & 4.29 & 3.83 & 11.79 & 3.29 & 43.48 \\
\hline $\begin{array}{c}\text { Third } \\
\text { Stage }\end{array}$ & 10.65 & 5.10 & 3.98 & 3.67 & 5.77 & 3.44 & 32.61 \\
\hline
\end{tabular}

\section{Conclusion}

After three stage experiments, the results suggested the suitable sentence length of each interface as shown in Table 1. According to the experiment result, we could also define that novice interface sentence would be changed into advanced speech sentence when it has been interrupted for 6 times, and advanced-novice interface would be changed into expert interface sentence when it has been interrupted for 5 times. In addition, the sentence of expert interface resulted in lowest degree of impatient, and required less operation time and mental workload than that of other interfaces. Therefore, drivers not only feel more convenience but also have a safer driving condition.

Acknowledgements. This research was sponsored by the Ministry of Economic Affairs, Taiwan, R.O.C. through project No. C352SN3100 conducted by ITRI.

\section{References}

1. Ministry of Transportation and Communications in Taiwan, 89th and 90th Road Safety Traffic Rules, http: / /motclaw.motc.gov.tw/

Law_ShowAl1.aspx?LawID=E0 $055087 \&$ Mode $=0 \&$ PageTit le $=\% \mathrm{E} 6 \%$ A2\%9D $\%$ 6\%96\%87\%E5\%85\%A7\%E5\%AE\%B9 (accessed December 13, 2011)

2. Jason, B., Calitz, A., Greyling, J.: From the Evaluation of an Adaptive User Interface Model. In: SAICSIT 2010 Proceedings of the 2010 Annual Research Conference of the South African Institute of Computer Scientists and Information Technologists (2010)

3. Global Road Safety Partnership, Speed management: a road safety manual for decisionmakers and practitioners, p. 6 (2008)

4. Transportation Engineering Online Lab Manual. Brake Reaction Time, http: / /www. webs1.uidaho.edu/niatt_labmanual/Chapters / geometricdesign/theoryandconcepts/BrakeReactionTime.htm (data accessed December 2, 2012) 